\section{altedical Socrecties.}

\author{
MEDICAL SOCIETY OF LONDON. \\ Saturday, March 17Th, 1855. \\ Dr. Snow, President.
}

\section{DR. STOCKER related the following}

CASE OF ANEURISMAL DILATATION OF THE AORTA.

In the spring of 1854, through the kindness of a medical friend, he was enabled to see the patient, who then complained of a constant uneasiness at the lower part of the chest, attended with a sensation of sinking, pain down both arms, and at the upper part of the left shoulder-blade; constant flatulence; no pain in swallowing; had never brought up any blood. The pulse at both wrists was full, equable, 90 ; intermitting, however, once or twice. On inspection, the chest was found to be well developed. On percussion, there was, anteriorly, on the right side, dulness, extending from the nipple downwards; on the left side the resonance was unimpaired. On auscultation, tubular breathing was audible on the right side, over the region where dulness on percussion existed; on the left side the re spiratory murmur was normal; posteriorly there was very slight modification of healthy respiration on either side. Heart: On percussion, there was slight increased dulness over the præcordial region. On auscultation, the sounds over the situation of the nistral and aortic valves were normal; there was, however, a slight diastolic bruit on tracing the sounds up the aorta over the space of an inch, about the situation of the right nipple. No pulsation, on examination by the finger, could be detected over the clavicle or sternum.

Previous History. - He had always enjoyed good health up to within the last twenty years, and in early life had used much muscular exertion.

After continuing in this state for some few months, he died in the summer of 1854, after having expectorated a slight quantity of blood for about two weeks previous to his decease.

Inspection, thirty hours after death. - The body showed slight evidences of incipient decomposition. On opening the thorax, the left lung was found to be adherent, by a few old pleuritic adhesions, to the parietes; as was also the right. On the left side there was also a small quantity of dirty-coloured serum; and on the right side there was about a pint and a half of fluid of a sero-sanguineous character. The left lung was found to be healthy ; the right, when cut into, gave out a large quantity of serous fluid, similar to that found in influenza, and its tissue was found to be somewhat compressed. The heart was dilated and hypertrophied, though more in proportion dilated; the valves on the left side somewhat thickened. The whole of the ascending aorta was excessively dilated, in some cases even forming pouches; on cutting into it, the inner and middle coats were found to be destroyed, their place being occupied by loose flakes of ossific matter, and in some places the outer coat had even been destroyed, and there was a slight aperture communicating with the lung on the right side. Where this had taken place the bronchus was rather flattened from pressure exerted on it by increased size of vessel. Throughout the entire length of the vessel, there were layers of fibrine deposited, evidently of some weeks' duration. The peritoneum on the surface of the liver was thickened and contracted, and the margin of the liver was rounded; on cutting into its substance, it was found to be contracted. The kidneys were congested and granular.

Mr. HenRy SMith related the particulars of

TWo CASES OF HERNIA PRESENTING INTERESTING FEATURES In the first instance he was called to a female, aged seventy. eight, who had a femoral hernia, which had become strangulated on the previous day, and had resisted attempts at reduction. When he saw her the symptoms were not very acute, but the hernia was very tense and irreducible. After a fair at tempt to return it, an immediate operation was recommended, but the patient would not consent to undergo it. The symptoms continued, but not in a very aggravated degree, and the woman remained alive for three weeks, nothing having passed by the bowels for this length of time. During the last week or ten days there had been continual vomiting shortly after taking a little fluid nourishment, and the pain had become very severe. The hernial tumours had somewhat increased in size. Mr. Smith thought that the interest connected with this case was in the circumstance of the patient living so long with a hernia in a state of strangulation. He attributed the duration of existence, and the comparative non-severity of symptoms, to the advanced age of the patient, when the actions of life have become feeble, and the changes consequent upon disease proportionately slow.

In the second case he had been called to see a male patient, aged sixty, who had suffered for two whole days from symptoms of strangulation of the intestine. He found an umbilical hernia of enormous magnitude hanging down to below the pubic region. The patient had carried the hernia in a bag for many years, and on two formeroccasions symptoms of strangulation had occurred and had lasted for many hours, but had been overcome by purgative medicines and other treatment. When the symptoms first occurred this last time, the hernia became increased in volume. Attempts had been made to return a portion of the intestine; cold was applied to the tumour, and purgatives and enemata were ordered by the gentleman who had attended him with such success on the previous occasions. Butnow they did not have any effect, and $\overline{M r}$. Smith recommended that an operation should be performed. This was delayed for several hours, at the request of the practitioner in attendance, who thought it possible that he might have the same suceess with treatment as on the former occasions. Injections of warm water, purgatives, and taxis, were again tried, but without any benefit, and sixty hours after strangulation had existed, when the patient was much exhausted, Mr. Smith cut down upon the neck of the tumour, made a small incision into the sac, and freely divided its neck, which was much thickened. The patient did not lose a teaspoonful of blood, and bore the operation very well, but he sank nine hours afterwards.

The interest connected with this case was the enormous magnitude of the tumour. It also showed the evil of delaying an operation for hernia until the patient has become exhausted but in the present instance an early operation was hardly justifiable, when the patient had on two previous occasions recovered from symptoms nearly as bad. He regretted that in neither case was he enabled to make an examination after death.

Mr. DENDy suggested that the first case related was one of incarcerated, and not strangulated, hernia; the symptoms of the first, in some cases, differing from those of the second only in degree. He related a case of irreducible hernia, in which the prominent symptoms of strangulation existed, but which gave way at the end of five weeks, under treatment by starvation, the patient having resolutely refused to be operated upon. Cases of irreducible umbilical hernia of large size were very common.

\section{Mr. Canton brought forward specimens of}

\section{DOUBLE INTRA-CAPSULAR FRACTURE OF THE CERVIX FEMIORIS,}

that is, a fracture of this part occurring on both sides of the body in one individual. The bones were removed from a subject which had been brought to the anatomical school of Charing-cross Hospital. The sex was female, and the age seventysix. On the right side, the fracture was close to the shalf, and quite ununited, and there also existed an oblique fracture of the trochanter-major extending from its summit, through it thickest part, and towards the lesser trochanter. Here firm ossific union had ensued. On the left side of the body, the neck of the femur was broken nearer to its head, and no attempt at reunion had taken place; the sharp margins of the cortex, however, had been almost completely rounded off in either fragment, and intestinal absorption had partially diminished the length of the cervix. Mr. Canton made some remarks upon the rarity of this double fracture, of which, unfortunately, no history could be obtained in the present instance. The medical certificate received with the body, stated the death to have resulted from " decay of nature." All the other bones were perfect.

\section{Dr. Rovth next read a paper on}

CERTAIN POINTS CONNECTED WITH THE PATHOLOGY, DIAGNOSIS, AND TREATMENT OF PNEUMONIA.

The author divided the subject into two parts-the first, relating to the general character of the disease, which he believed adynamic; its diagnosis, and the theoretical indications against treatment by bloodletting. The second, relating to the actual results of different plans of practice. The first part only was considered this evening.

I.- The disease gave evidence that the blood especially was affected, being, in its first stages, unusually fluid, subsequently hyperinosed. He showed that-first, the effect of thinning or impoverishing the blood was to produce pneumonia. The experiments of Magendie were quoted in confirmation of this 
point, in which, by injecting defibrinized blood, pus, or putrid matter in the blood, pneumonia was almost invariably produced. 'The production of fibrine, in the course of the disease, was a secondary result, due to increased respiration. The blood was also chemically deficient in chlorides, the first effect of which was also to produce unusual fluidity of the blood, and general dropsy as a consequence; and the second, to lead to a deposition of the fibrine, which, being increased in quantity, was no longer capable of being held in solution, both from the absence of the chlorides, as also of the alkaline phosphates, which derived their soda base from the chloride of sodium. The occurrence of pneumonia in cases of wounds, or after surgical operations, was also explained by the absorption of putrid matters or pus, and the necessary fluidity of the blood. Lastly, the causes of pneumonia were statistically shown to be very frequently those which brought about fluidity of the blood. Pneumonia was also most fatal, in proportion as it was of a low type, and in typhus there was also a deficiency of chlorides. From these facts Dr. Routh concluded that the disease was essentially adynamic.

II.-In regard to the diagnosis, Dr. Routh showed, from the Registrar-General's Reports, that the disease called pneumonia, was made to include a variety of other diseases of a totally different character. Taking the years 1840 to 1844 , inclusive, for London, the deaths were, from pneumonia, one-half in number those registered from phthisis, and eight times those from bronchitis; and for all England, for the years 1847 and 1848, they were nearly equal to those from phthisis, and twice as numerous as those from bronchitis. Capillary bronchitis was not even mentioned -a result altogether opposed to hospital experience. Pneumonia was very often confounded with simple pulmonary congestion, capillary bronchitis, and a particular variety of pleuritis. Dr. Routh pointed out the differences of these three diseases, by the physical signs, general symptoms, and peculiar characteristics of the dyspnoea, cough, and expectoration, dwelling particularly on the so-called fine crepitation, upon which so much stress was laid in the present day. Some allusion was also made to œdema of the lungs, and pulmonary apoplexy. In regard to the prognosis, Dr. Routh believed that, within favourable ages, the disease was generally curable by any prudent treatment, although more fatal, as a rule, in Great Britain, because more than fifty-three per cent. were generally complicated cases.

III. - In regard to the theory of the treatment, bloodletting has been recommended, because-

1st. - It was said to diminish the amount of fibrine; but it had been shown by Andral, Gavarret, Simon, and others, that in pneumonia, rheumatism, peritonitis, \&c., the fibrine was not diminished till after the fourth or fifth venesection; but even the advocates of bloodletting generally disapproved of such heroic renesections.

2nd. - It was said to diminish the fever, and the severity of the general symptoms. Dr. Routh admitted this; but then it must be carried to great excess, and the secondary consequences, debility, and a long period of convalescence, were the results.

3rd.- - It was said to diminish the severity of the local symptoms. This was the exception, not the rule, and opposed to the experience of Grisotte, Todd, Chomel, and Ragori; and Laennec even went so far as to say that, in intense pneumonia, it aggravated the symptoms. Bloodletting, perhaps, shortened the duration of the febrile excitement, but lengthened the convalescence. Dr. Routh, therefore, concluded that the treatment by bloodletting was, pathologically and physiologically, unphilosophical.

A discussion ensued, in which Dr. Henry Lee, Dr. Webster, Mr. Dendy, and Dr. Willshire took part.

\section{NORTH LONDON MEDICAL SOCIETY.}

February 14th, 1855.

Mr. Qualn, Prestoent, in the Chatr.

\section{Mr. FinLITER related the following case of}

PROLAPSUS OF THE LOWER END OF THE ILIUM, FOLLOWING TUBERCULAR ULCERATION OF THE SMALL INTESTINE AND IrIO-C

C. B- aged four years, one of a family of six children, (of whom two show signs of a scrofulous diathesis,) was before the attack apparently in robust health, excepting the occasional presence of worms in the motions and a slight tendency to diarrhœea.
On the morning of Jan. 31st, 1855, ten days before death, the patient was seized suddenly with vomiting, and twice during the day she passed small jelly-like motions, mixed with blood; the following day the belly became painful and began to swell; the vomiting increased in urgency, and no evacuation followed the use of purgatives and enemata.

On Feb. Tth, the child was admitted into the Marylebone Infirmary. He was very restless, continually asking for drink, with an anxions expression of face, and a moderately full but rapid pulse; the body was swollen but not tense, tympanitic, and tender only on strong pressure ; the skin was hot; the tongue slightly furred and moist. A purgative dose of calomel was given, and repeated on the following morning. A castor oil injection was ordered, but before evening the bowels began to act, the vomiting ceased, and the child became quieter. On the 9 th several small liquid motions, not containing blood, were passed. In the evening she became much lower, and died early on the morning of the 10th.

Post-mortem examination, two days after death.-On opening the abdomen, the intestines were found distended, and their opposed surfaces slightly reddened; no lymph was found in the abdominal cavity on removing the intestines and opening the cæcum, which formed a large, somewhat resistant mass ; it was found that a coil of the ilium, six inches and a half in length was lying coiled upon itself within it; this had descended through the ulcerated ilio-cæcal valve, and being invaginated, not less than thirteen inches of the lower end of the ilium were contained in the cecum. The uniform dark-red colour and swollen condition of the prolapsed intestine, together with the distended state of the bowels above were such as to indicate complete strangulation, but the escape of a small quantity of fluid frees from the distal extremity of the coil, and the diar. rhoea which set in two days before death, showed that the canal had become in some degree pervious. The mucous mem. brane of the small intestine was the seat of numerous ulcers; they were most numerous in the ilium, in some instances corresponding with Peyer's patches, in others extending transversely round the intestine, and destroying the valvulæ conniventes; the prolapsed portion also exhibited several ulcers. The mesenteric glands were much enlarged, of pale colour, and natural consistence. At the posterior and upper part of the lower lobe of the left lung were two deposits of yellow tubercle about the size of a pea. The other organs of the body were healthy.

\section{Thursday, March 1 ist.}

\section{Mr. Filliter read a paper on}

FIBRO-PLASTIC TUMOURS OF THE DURA IIATER.

The patient was admitted into the Marylebone Infirmary on February 4th, in a state of maniacal excitement, which had come on suddenly the day before, and which terminated in coma and death the following evening. Whilst under observation she was unable to speak, but the face was frequently distorted with grimaces, and the legs were in constant motion; the left arm was rigid, and insensible to pinching. She was said by her neighbours to be an eccentric character, and had been noticed to stagger a little on walking for about a week previously. In other respects she appeared in her usual health until the date of the attack. She had been living in much privation with an aged brother, who is now in the Infirmary in a state of partial imbecility. At the post-mortem, on removing the dura mater from the posterior part of the brain, two tumours were found attached to its under surface, and were readily withdrawn from cavities which they occupied in each hemisphere. The larger tumour was smooth, shining, globular, and elastic, and weighed five drachms and a half, the diameter of its section being one inch and a quarter; the colour of the cut surface was an almost uniform greyish white, speckled with opaque granules; the consistence was friable, resembling that of a pear. The smaller tumour was about the size of a marble, and resembled the other, with the exception of being gritty on section. The cerebral substance generally was unusually firm. In the right posterior pillar of the fornix was a hard, grey, translucent nodule. Each hippocampus, but especially the right, was remarkably indurated, its grey matter exposed on section being almost cartilaginous; the cerebellum also was firmer than natural. A bout two ounces of coagulable serum were found at the base of the brain. The other organs of the body were healthy. On microscopic examination, the larger tumour was found to consist of fibrous tissue and fibro. plastic cells, which were not materially altered by the addition of acetic acid; there were also free nuclei. The smaller tumour was similar in structure, but contained numerous calcareous spiculæ, and bodies in which a laminated fibrous basis was de- 